\title{
Emergências hipertensivas
}

\author{
Hypertensive emergencies
}

Monique E. Cardoso*

\section{Resumo}

A elevação da pressão arterial é frequente no ambiente de terapia intensiva, podendo ser o motivo da admissão de determinados pacientes ou se desenvolver em pacientes que já se encontram internados. Pode se manifestar como uma emergência, requerendo pronta-redução dos níveis pressóricos com agentes intravenosos, ou como uma urgência, na qual o controle pressórico pode ser feito de forma mais gradual com agentes orais. $\mathrm{O}$ fato que diferencia essas duas situações é a presença de lesões de órgãos-alvo, encontradas nas emergências hipertensivas, levando à manifestações principalmente neurológicas (encefalopatia hipertensiva, acidente vascular cerebral isquêmico ou hemorragia intracerebral), hematológicas (hemólise microangiopática), cardiovasculares (insuficiência ventricular esquerda aguda, isquemia e infarto miocárdicos e dissecção aórtica), ou renais (oligúria, azotemia). As emergências hipertensivas podem também surgir em pós-operatório ou estar associadas à elevação de catecolaminas e eclampsia.
A abordagem inicial do paciente inclui a anamnese e exame físico, em busca de informações, como presença de hipertensão arterial prévia, presença de outras comorbidades e manifestações relacionadas a lesões de órgãos-alvo. Exames complementares, laboratoriais e de imagem também são necessários, em busca dessas lesões.

Devido à morbidade e mortalidade associada a estas situações, a pressão arterial deve ser rapidamente controlada com agentes endovenosos. A terapia inicial tem o objetivo de reverter a lesão de órgãos-alvo e não retornar a pressão arterial ao normal. Posteriormente podem ser introduzidos agentes orais.

A escolha e os objetivos do tratamento são baseados em consensos e devem ser guiados pela etiologia da hipertensão, extensão das lesões de órgãos-alvo e perfil hemodinâmico individual do paciente.

Descritores: Hipertensão; Emergências; Traumatismo múltiplo. 


\section{Abstract}

Elevated blood pressure is common in the intensive care unit setting and may be the reason for the admission of certain patients or develop in patients who are already hospitalized. It can manifest as an emergency, requiring prompt reduction of blood pressure with intravenous agents, or as urgency, where blood pressure control can be done more gradually with oral agents. The fact that differentiates these two situations is the presence of target organ damage, found in hypertensive emergencies, leading to findings mainly neurological (hypertensive encephalopathy, ischemic stroke or intracerebral hemorrhage), hematologic (microangiopathic hemolysis), cardiovascular (acute left ventricular failure, myocardial ischemia and infarction and aortic dissection), or renal (oliguria, azotemia). The hypertensive emergencies can also arise in postoperative or associated with elevation of catecholamines and eclampsia.

The initial approach includes the patient's history and physical examination, looking for information such as the presence of preexisting hypertension, prior comorbidity and manifestations related to target organ damage. Additional exams are also needed, such as laboratory tests and imaging studies in search of target organ damage.

Because of the morbidity and mortality associated with these situations, blood pressure should be controlled quickly with intravenous agents. Initial therapy aims to reverse the target organ damage and does not return to normal blood pressure. Subsequently oral agents can be introduced.

The choice and the goals of treatment are based on consensus and should be guided by the etiology of hypertension, extent of target organ damage and hemodynamic profile of the individual patient.

Keywords: Hypertension; Emergencies; Multiple trauma.

\section{Introdução}

Os pacientes internados em unidade de tratamento intensivo que se apresentam com pressão arterial elevada podem apresentar uma hipertensão que leva a dano iminente de órgãos-alvo, a emergências hipertensivas, ou a uma hipertensão transiente mais benigna, denominada urgência hipertensiva. ${ }^{1}$ Devido à presença de lesões de órgãos-alvo evidenciada nos casos de emergências hipertensivas, o médico intensivista deve estar apto a reconhecer as manifestações clínicas e instalar prontamente o tratamento, para diminuir a morbidade e mortalidade associadas a estas complicações. Neste capítulo serão descritas estas situações, com enfoque na classificação, fisiopatologia, abordagem do paciente, fármacos disponíveis para o tratamento e as diversas formas de apresentação das lesões orgânicas.

\section{Classificação}

É denominada crise hipertensiva qualquer situação que leve à elevação da pressão arterial. ${ }^{1}$ Algumas circunstâncias, denominadas urgências e emergências hipertensivas, são ameaçadoras à vida e requerem imediato controle. ${ }^{1,2}$ Podem ocorrer em pacientes com piora de uma hipertensão crônica, em portadores de formas secundárias de hipertensão ou em pacientes sem doença hipertensiva previamente conhecida. ${ }^{1}$

Emergência hipertensiva significa elevação da pressão arterial caracterizada por quadro clínico grave, progressiva lesão de órgãos-alvo e risco de morte. ${ }^{3}$ Estas lesões de órgãos-alvo podem ser neurológicas, miocárdicas, vasculares, hematológicas ou renais ${ }^{1}$ e requerem redução imediata (porém controlada) da pressão arterial. ${ }^{2}$ Devem ser utilizados medicamentos aplicados por via parenteral. ${ }^{3}$

Urgência hipertensiva é uma elevação crítica da pressão arterial com estabilidade clínica, sem comprometimento de órgãos-alvo, ${ }^{3}$ porém com grande potencial de lesão orgânica se não for controlada. Nos pacientes que se apresentam hipertensos, assintomáticos, sem lesões de 
órgão-alvo não há benefício de redução rápida da pressão arterial, ${ }^{1}$ sendo que a hipertensão pode ser tratada mais lentamente. ${ }^{2}$ Nestes casos, devem ser utilizados medicamentos por via oral, buscando-se redução da pressão arterial em até 24 horas. Pacientes que cursam com urgência hipertensiva estão expostos a maior risco futuro de eventos cardiovasculares comparados com hipertensos que não a apresentam, fato que evidencia o seu impacto no risco cardiovascular dos indivíduos. ${ }^{3}$

Nas crises hipertensivas a elevação da pressão arterial tende a ser grave, com pressão arterial diastólica (PAD) maior que $120 \mathrm{mmHg}$. Os níveis de pressão arterial sistólica (PAS), PAD ou pressão arterial média (PAM) não diferenciam as categorias, mas sim a presença ou ausência de lesão de órgão-alvo aguda e progressiva. ${ }^{1}$

Hipertensão acelerada ou maligna são termos antigos baseados em achados oftálmicos como retinopatia exsudativa, hemorragias retinianas ou papiledema, ${ }^{1}$ ilustrados na figura $1 .{ }^{4}$ A hipertensão acelerada pode ser uma urgência ou emergência. ${ }^{1}$

\section{Incidência}

Não há dados confiáveis a respeito da incidência de emergências hipertensivas. Nos Estados Unidos a hipertensão é o diagnóstico primário em mais de 500.000 admissões hospitalares. Os pacientes com hipertensão essencial que se apresentam nas salas de emergência com crises hipertensivas tendem a ser homens jovens e com baixo nível socioeconômico, conscientes do diagnóstico de hipertensão e em uso de medicação, porém com má-adesão. ${ }^{1}$

Menos de 1\% dos pacientes com hipertensão progridem para uma fase acelerada/ maligna, provavelmente devido ao tratamento mais disseminado da hipertensão. ${ }^{2}$ Qualquer doença hipertensiva pode se manifestar como crise. ${ }^{1}$ Algumas causas como feocromocitoma e hipertensão renovascular o fazem com a mesma frequência do que hipertensão primária. Porém, como a hipertensão é de causa desconhecida em $90 \%$ dos pacientes, a maioria das crises hipertensivas ocorre no contexto da hipertensão primária pré-existente. $^{2}$

\section{Fisiopatologia}

A maioria dos órgãos controla a quantidade de fluxo sanguíneo recebida através de um mecanismo denominado autorregulação. ${ }^{1}$ Desta forma, o fluxo sanguíneo é determinado pela pressão de perfusão do órgão e pela resistência vascular do mesmo. Pequenas artérias e arteríolas contraem ou dilatam em resposta a fatores miogênicos locais que atuam no endotélio e respondem a gradientes de pressão transmural. Uma diminuição da pressão de perfusão leva à vasodilatação, enquanto um aumento da pressão de perfusão leva à vasoconstrição e limita o dano induzido pela pressão. ${ }^{1}$

$\mathrm{Na}$ maioria dos pacientes com crise hipertensiva a anormalidade fisiopatológica é um aumento da resistência vascular sistêmica e

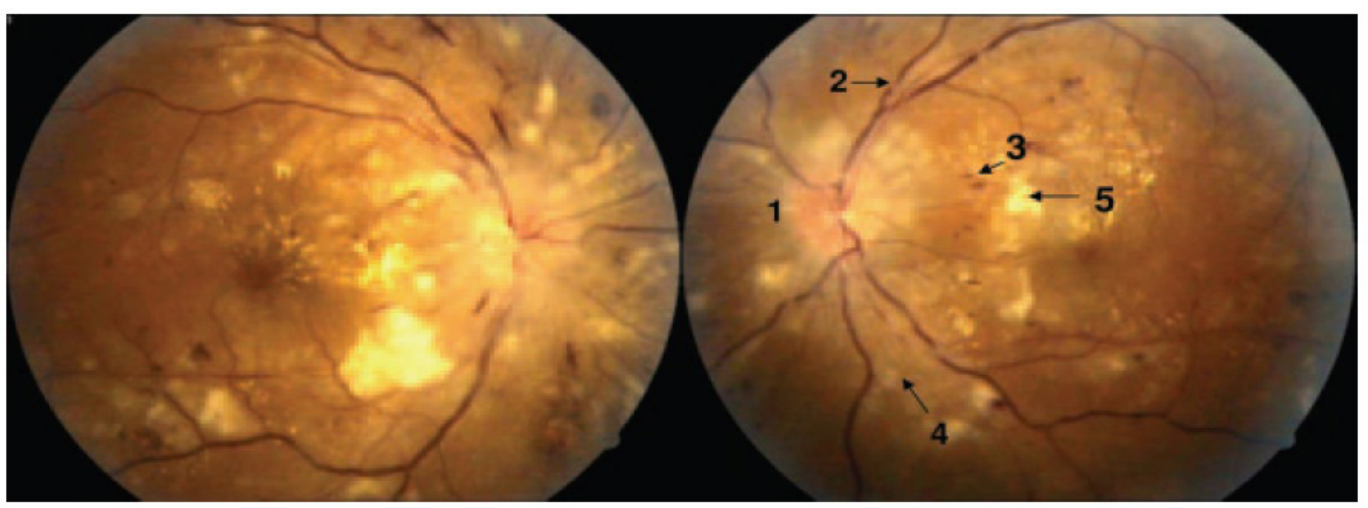

Figura 1. Fundoscopia ilustrando a retinopatia hipertensiva grau IV Pode ser visto papiledema (1), cruzamento arteriovenoso (2), hemorragias em chama de vela (3), exsudatos moles (4) e duros (5). ${ }^{4}$ 
não aumento do débito cardíaco. O aumento da resistência vascular eleva a pressão arterial, prejudica a autorregulação local e leva à isquemia orgânica. ${ }^{1}$

A circulação cerebral pode manter a perfusão com alterações da PAM de 60-120 mmHg em pacientes normotensos e entre 110-180 mmHg em pacientes hipertensos, ${ }^{2}$ sendo que quando a PAM excede o alcance autorregulatório pode haver perda da autorregulação ${ }^{1}$ e hiperperfusão. ${ }^{2}$ Este desvio que ocorre em pacientes hipertensos resulta de espessamento estrutural das arteríolas como uma adaptação à elevação crônica da pressão. Em pacientes previamente normotensos as alterações na autorregulação ocorrem em uma PAM em torno de $120 \mathrm{mmHg}$ (160-100 mmHg), enquanto em hipertensos ocorre em uma PAM em torno de $180 \mathrm{mmHg}$ (260-140 mmHg). Os vasos contraídos não conseguem resistir a esta pressão e dilatam. A vasodilatação começa de uma maneira irregular, primeiro em áreas com menos tônus muscular e em seguida difusamente, com vasodilatação generalizada. Isso leva à hiperperfusão do cérebro sob uma alta pressão, causando vazamento de fluido para o tecido perivascular, resultando em edema cerebral e síndrome de encefalopatia hipertensiva. ${ }^{2} \mathrm{~A}$ figura 2 ilustra os achados neurológicos evidenciados através da tomografia computadorizada e ressonância magnética cerebrais. ${ }^{4}$ Além do aumento de permeabilidade também ocorre ativação da cascata da coagulação e depósito de plaquetas e fibrina. Com elevações importantes da pressão arterial há dano endotelial e necrose fibrinoide das arteríolas. Este processo resulta em isquemia e liberação de agentes vasoativos adicionais, gerando um ciclo vicioso de injúria. Ocorre natriurese pressórica, levando à depleção de volume e estímulo a liberação de substâncias vasoconstritoras pelo rim. Estes mecanismos coletivos levam à hipoperfusão de órgãos-alvo, isquemia e disfunção, manifestando-se como emergências hipertensivas. ${ }^{5}$

A velocidade de aumento é mais importante do que o valor em si para levar a dano vascular. Porém, na prática, todos os pacientes com PAD maior que $130 \mathrm{mmHg}$ devem ser tratados, alguns mais rapidamente com medicamentos parenterais e outros mais lentamente com medicações orais. ${ }^{2}$

Quando a pressão de perfusão cai abaixo do mecanismo autorregulatório pode ocorrer isquemia e infarto, sendo que a tolerância varia entre os indivíduos. Idosos e hipertensos toleram hipertensão devido ao desvio para cima do mecanismo de autorregulação cerebral, porém têm baixa tolerância à hipotensão. Já pacientes sem hipertensão prévia podem apresentar vasculite aguda, hemorragia subaracnoidea, angina instável ou eclampsia com menores valores de pressão arterial. ${ }^{1}$

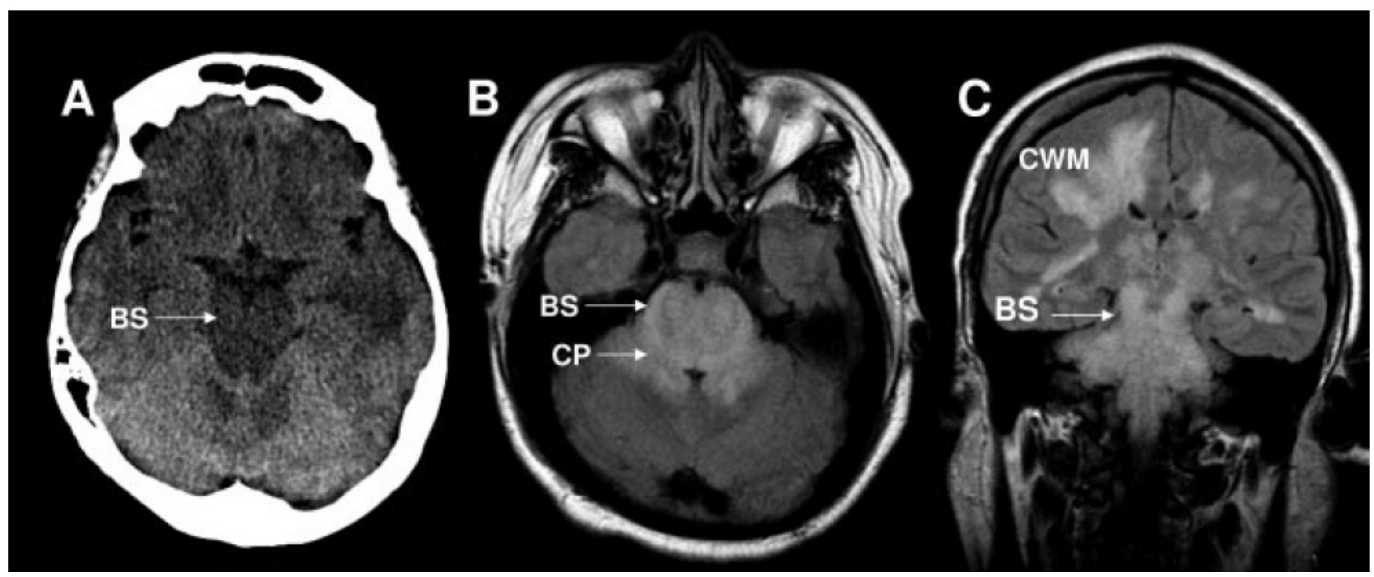

Figura 2. Imagens do cérebro ilustrando a encefalopatia hipertensiva

Podem ser vistas hipodensidades no tronco cerebral e substância branca cerebral compatíveis com edema (A). Em B e C são vistos cortes de ressonância magnética evidenciando hiperintensidade no tronco cerebral, pedúnculo cerebelar e substância branca cerebral. ${ }^{4}$ 


\section{Abordagem do paciente}

Alguns pacientes podem estar assintomáticos apesar de elevação importante da pressão arterial e dano extenso a órgãos-alvo. Se não tratado, o paciente pode rapidamente evoluir para óbito devido ao dano cerebral ou mais gradualmente devido ao dano renal. ${ }^{2}$

Uma breve história e exame físico devem avaliar o grau de lesão de órgão-alvo e excluir causas óbvias de hipertensão secundária.

A abordagem ao paciente deve incluir anamnese, exame físico e exames complementares, da seguinte forma:

\section{Anamnese}

- Presença de HAS prévia; ${ }^{1}$

- Presença de outras doenças significantes; ${ }^{1}$

- Uso de medicações; ${ }^{1}$

- Adesão ao tratamento; ${ }^{1}$

- Uso de drogas ilícitas; ${ }^{1}$

- Sintomas de lesões de órgãos-alvo, como cardíacos, neurológicos ${ }^{1}$ e renais (oligúria, azotemia). ${ }^{2}$

\section{Exame físico}

- Avaliação da pressão arterial em ambos os braços em posição de pé e sentada; ${ }^{1}$

- Excluir pseudo-hipertensão pela manobra de Osler (insuflar o manguito acima da pressão sistólica; artéria radial ainda palpável na ausência de pulso indica artéria enrijecida e pressão arterial superestimada);

- Exame oftálmico em busca de hemorragias, exsudatos e papiledema. ${ }^{1}$ A fundoscopia é fundamental, pois a presença de retinopatia avançada está associada à presença de disfunção microvascular disseminada e injúria renal $;^{5}$

- Ausculta pulmonar;

- Avaliação cardíaca: impulso apical pro- eminente, aumento da área cardíaca, insuficiência cardíaca congestiva; ${ }^{2}$

- Avaliação abdominal em busca de massas ou sopros; ${ }^{1}$

- Palpação de pulsos periféricos; ${ }^{1}$

- Busca de sinais neurológicos compatíveis com isquemia (alteração do estado mental, cefaleia, náusea e vômitos associados a déficit neurológico focal). ${ }^{1}$

\section{Exames complementares}

- Dosagem de eletrólitos, ureia, creatinina, leucometria total e diferencial, marcadores de lesão miocárdica (se necessário); ${ }^{1}$

- Investigação de hemólise microangiopática, detectada na presença de plaquetopenia associada a aumento de LDH e presença de esquizócitos. É importante fazer este diagnóstico, pois está associado a dano renal irreversível; ${ }^{5}$

- Análise de urina em busca de proteinúria e hematúria microscópica; ${ }^{5}$

- Ecocardiograma: avaliação da função sistólica e diastólica, importante em pacientes com edema pulmonar; ${ }^{5}$

- Radiografia de tórax: avaliar tamanho da aorta, cardiomegalia; ${ }^{1}$

- Avaliação do débito urinário;

- Eletrocardiograma: avaliação de isquemia; ${ }^{1}$

- Avaliação do sistema nervoso central: tomografia computadorizada de crânio sem contraste deve ser realizada em pacientes com sintomas neurológicos para excluir hemorragia cerebral ou efeito de massa; a ressonância magnética de crânio é mais sensível do que a tomografia computadorizada para detecção de isquemia, edema e alterações na substância branca parieto-occipital (síndrome de leucoencefalopatia pos- 
terior, associada à encefalopatia hipertensiva). A identificação de eventos vasculares cerebrais isquêmicos ou hemorrágicos é importante devido ao diferente manejo da hipertensão arterial, com alvos diferentes de pressão arterial em relação à encefalopatia hipertensiva; ${ }^{1}$

- Em suspeita de dissecção aórtica, deve ser realizada angiotomografia ou angiorresonância de aorta com contraste. Embora o ecocardiograma transesofágico tenha alta sensibilidade e especificidade para dissecção, deve ser realizado apenas após alcance do controle pressórico. ${ }^{5}$

\section{Tratamento}

O tratamento das emergências hipertensivas não é baseado em estudos randomizados e controlados de grande porte, já que a maioria dos estudos não foi realizado desta forma. Uma revisão sistemática não encontrou também evidências apoiando um anti-hipertensivo em vez de outro. A decisão entre medicamentos orais ou parenterais depende de diversos fatores, como presença de emergência hipertensiva, necessidade de um efeito rápido, necessidade de redução rápida da pressão arterial, necessidade de um agente de curta duração, risco de hipotensão, presença de doença aterosclerótica cardíaca, presença de hipertensão renovascular, doença cerebrovascular ou desidratação. ${ }^{1}$ Para pacientes sem perigo imediato, o tratamento oral pode ser utilizado. ${ }^{2} \mathrm{O}$ quadro 1 descreve as principais situações que requerem tratamento imediato da hipertensão arterial. ${ }^{2}$

A monitorização invasiva da pressão arterial pode ser necessária, caso altas doses de vasodilatador venoso sejam exigidas ou quando por qualquer motivo técnico a pressão não invasiva não esteja confiável, ${ }^{6}$ já que uma dose levemente excessiva pode diminuir a pressão arterial abruptamente para níveis que induzem choque. ${ }^{7}$ Devem ser realizados também monito- rização eletrocardiográfica contínua, oximetria de pulso e obtenção de um acesso venoso para administração de medicamentos parenterais. ${ }^{6}$ Podem ser necessárias medidas como intubação, controle de convulsões e manutenção do débito urinário. ${ }^{1}$

A terapia inicial deve reverter o dano de órgãos-alvo e não retornar a PA ao normal. ${ }^{1}$

\section{Quadro 1. Situações que requerem rápido trata- mento da hipertensão arterial}

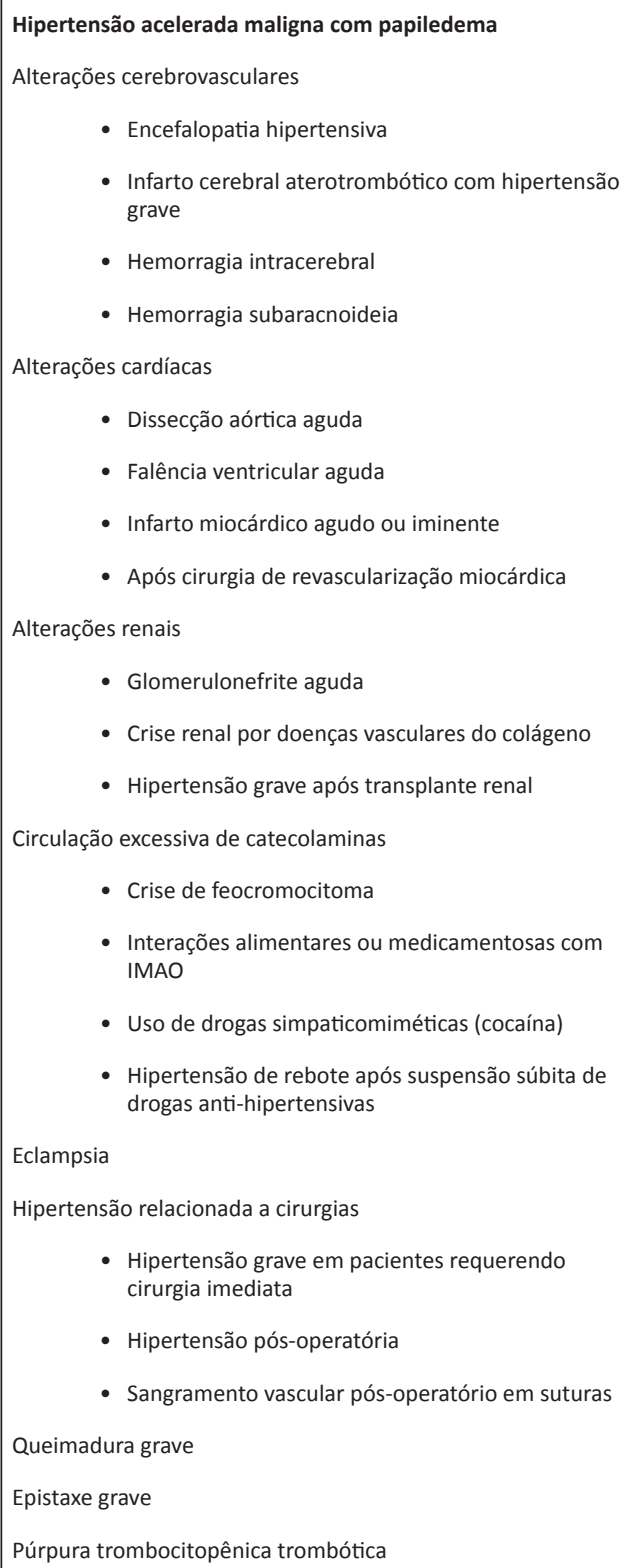

- Infarto miocárdico agudo ou iminente

- Após cirurgia de revascularização miocárdica

Alterações renais

- Glomerulonefrite aguda

- Crise renal por doenças vasculares do colágeno

- Hipertensão grave após transplante renal

Circulação excessiva de catecolaminas

- Crise de feocromocitoma

- Interações alimentares ou medicamentosas com IMAO

- Uso de drogas simpaticomiméticas (cocaína)

- Hipertensão de rebote após suspensão súbita de drogas anti-hipertensivas

Eclampsia

Hipertensão relacionada a cirurgias

- Hipertensão grave em pacientes requerendo cirurgia imediata

- Hipertensão pós-operatória

- Sangramento vascular pós-operatório em suturas

Queimadura grave

Epistaxe grave

Púrpura trombocitopênica trombótica

O quadro acima descreve as principais circunstâncias 
Como a circulação cerebral é mais sensível à isquemia, o limite inferior da autorregulação cerebral para cada paciente determina o objetivo inicial. Este limite inferior é aproximadamente $25 \%$ menor do que a PAM inicial ou PAD na faixa de 100-110 mmHg. ${ }^{1}$ Um tratamento inicial razoável consiste em diminuir a PAM em 25\% em 1-2 horas e posteriormente atingir um alvo em torno de 160/110 mmHg em 2-6 horas, ${ }^{8} \mathrm{com}$ agentes que diminuem a resistência vascular periférica, considerando a história médica, eventos iniciais e evolução das lesões de órgãos-alvo. ${ }^{1} \mathrm{~A}$ pressão arterial pode ser reduzida ao normal nas próximas 24 a 48 horas, com redução gradual da administração da medicação parenteral. ${ }^{6}$

Rápida correção da pressão arterial abaixo da faixa autorregulatória pode levar à redução da perfusão, com isquemia e infarto. Assim, embora a pressão arterial deva ser diminuída nestes pacientes, esta diminuição deve ser lenta e controlada, para prevenir hipoperfusão orgânica. Uma importante consideração antes de iniciar a terapia intravenosa é a determinação do estado volêmico do paciente. Devido à natriurese pressórica, muitos pacientes que se apresentam com emergências hipertensivas se encontram depletados de volume, sendo que a restauração do volume intravascular com salina intravenosa irá restaurar a perfusão orgânica e prevenir uma queda abrupta da pressão arterial com início das medicações. ${ }^{5}$

Pacientes com insuficiência cardíaca aguda, isquemia miocárdica ou dissecção aórtica requerem abordagem mais agressiva. ${ }^{1}$ Por outro lado, reduções excessivas da PA podem precipitar isquemia cerebral, coronariana e renal. ${ }^{8}$

Após a estabilidade do paciente, devem ser realizados exames diagnósticos adicionais. Um regime oral pode ser iniciado. ${ }^{1}$

Os principais medicamentos endovenosos utilizados no tratamento das emergências hipertensivas em nosso meio são os seguintes:

\section{Vasodilatadores diretos}

Nitroprussiato: atua dilatando arteríolas e vênulas, diminuindo a pré e pós-carga e a de- manda de oxigênio do miocárdio. Seu efeito é mediado pelo GMP cíclico intracelular e óxido nítrico em um mecanismo endotelial. Tem início e término de ação rápidos. ${ }^{1}$ Consequentemente, a cessação abrupta da infusão causará aumento pressórico quase imediato. A despeito de seu perfil farmacocinético e farmacodinâmico ideal, o nitroprussiato possui limitações, principalmente quando usado de modo prolongado (> 24-48 horas) e/ou em altas doses (> $2 \mu \mathrm{g} / \mathrm{kg} /$ min). ${ }^{6}$ É rapidamente decomposto em cianeto, que é convertido em tiocianato no fígado. Em altas doses pode haver toxicidade aguda por acúmulo de cianeto, ${ }^{1} \mathrm{o}$ que pode se manifestar com acidose metabólica, confusão mental, coma, encefalopatia, cefaleia e bloqueio cardíaco inexplicado. ${ }^{6} \mathrm{O}$ metabólito tiocianato pode se acumular em insuficiência renal aguda ou crônica e seus níveis devem ser monitorados. É removido com diálise. ${ }^{1}$ Devido à potência e à rapidez de ação do nitroprussiato, este é o agente de escolha para tratamento de hipertensão ameaçadora à vida. ${ }^{7}$ Diminui o fluxo sanguíneo cerebral e aumenta a pressão intracraniana, efeito que é desvantajoso em pacientes com encefalopatia hipertensiva e após acidente vascular cerebral. ${ }^{5}$

Nitroglicerina: dilata preferencialmente as veias, via GMP cíclico. A pressão diastólica esquerda é reduzida sem queda no volume sistólico ou débito cardíaco, porém a pressão arterial diminui modestamente. Aumenta o fluxo em colaterais coronarianas e melhora o fluxo sanguíneo epicárdico, sendo útil em pós-operatório de cirurgia de revascularização miocárdica, isquemia coronariana e em insuficiência cardíaca. Deve ser evitada em pacientes com aumento da pressão intracraniana, estenose aórtica e cardiomiopatia hipertrófica. ${ }^{1}$ É contraindicada nos casos de infarto de ventrículo direito e nos pacientes que usaram sildenafil nas últimas 24 horas. ${ }^{6}$ Por ser menos potente que o nitroprussiato, este agente fica reservado para pacientes com síndromes isquêmicas miocárdicas. ${ }^{8}$

Hidralazina: é um vasodilatador direto que aumenta o débito cardíaco e a frequência cardíaca. ${ }^{1}$ Tem efeito prolongado e imprevisível, além 
da impossibilidade de titular seu efeito hipotensor. ${ }^{5}$ É contraindicada nos casos de síndromes isquêmicas miocárdicas agudas e de dissecção aguda de aorta por induzir ativação simpática, com taquicardia e aumento da pressão de pulso. ${ }^{3}$ Geralmente é usada nas emergências hipertensivas em gestantes, devido a sua segurança comprovada para o feto. Tem como efeitos colaterais a possibilidade de induzir doenças reumatológicas ou imunológicas, sendo sua suspensão geralmente curativa ${ }^{6}$

\section{Betabloqueadores}

Muitos podem ser administrados via parenteral, como propranolol, metoprolol, esmolol e labetalol. Levam à diminuição do consumo miocárdico de oxigênio e melhora da hemodinâmica coronariana em pacientes com doença arterial coronariana. ${ }^{1}$ São contraindicados em pacientes com insuficiência ventricular descompensada, portadores de doença pulmonar obstrutiva crônica (DPOC) descompensada ou asma, vasculopatia periférica grave e bloqueios atrioventriculares. ${ }^{6} \mathrm{O}$ esmolol, devido a sua ação de curta duração, é útil quando há a preocupação quanto aos efeitos colaterais dos betabloqueadores. ${ }^{8}$

\section{Antagonistas dos canais de cálcio}

Verapamil: promove alentecimento da condução atrioventricular e efeito inotrópico negativo, com início rápido de ação e baixa incidência de efeitos colaterais. Pode ser dado em bolus repetidos ou em infusão contínua. ${ }^{1}$

Diltiazem: tem efeito intermediário entre os diidropiridínicos e verapamil. É amplamente utilizado para controle de frequência em fibrilação atrial, mas não como agente antihipertensivo primário. ${ }^{1}$

Esses dois agentes podem ser utilizados na impossibilidade de uso de betabloqueador devido a alguma contraindicação. São contraindicados na presença de bloqueios atrioventriculares e na presença de falência ventricular. ${ }^{6}$

\section{Inibidores alfa-adrenérgicos}

Fentolamina: é o único alfa-antagonista disponível para administração intravenosa. Seu uso é reservado para estados associados a excesso de catecolaminas, como feocromocitoma, hipertensão de rebote ou ingestão de drogas. $\mathrm{O}$ efeito hipotensor está associado à taquicardia reflexa. A vantagem é seu efeito específico no feocromocitoma. As desvantagens incluem dor abdominal, vômitos, diarreia, taquicardia, tonteira e arritmias. ${ }^{1}$

\section{Diuréticos}

Pacientes com hipertensão no pósoperatório, disfunção cardíaca ou edema pulmonar podem requerer diuréticos. Além disso, muitos anti-hipertensivos parenterais podem causar retenção de fluidos. Diuréticos de alça ajudam a controlar o volume intravascular, mantêm o débito urinário e previnem resistência à terapia anti-hipertensiva. Podem ser administrados em bolus ou em infusão lenta. ${ }^{1}$

A tabela 1 descreve os principais anti-hipertensivos parenterais utilizados no tratamento das emergências hipertensivas.

\section{Tratamento das emergências hipertensivas específicas}

\section{Emergências cardiovasculares}

\section{Insuficiência ventricular esquerda aguda}

Os agentes de escolha são nitroprussiato e nitroglicerina para diminuir a congestão pulmonar através da diminuição da resistência vascular sistêmica e da PAM. Promovem ainda diminuição da pré-carga, sendo utilizados em edema pulmonar junto com diuréticos. ${ }^{1}$

\section{Isquemia miocárdica e infarto}

O tratamento da hipertensão é uma das formas de restaurar a perfusão miocárdica. Como o nitroprussiato pode desviar o fluxo de áreas pós-estenóticas, a nitroglicerina é preferida. Betabloqueadores também promovem diminuição da pressão sistêmica, da demanda 
miocárdica de oxigênio, da frequência cardíaca e da pressão arterial. ${ }^{1}$ Deve-se reduzir a pressão arterial em $30 \%$, já que reduções mais bruscas podem diminuir também a perfusão coronariana. ${ }^{6}$ É importante ressaltar que hipertensão não controlada (PAS > $180 \mathrm{mmHg}$ ou PAD > $110 \mathrm{mmHg}$ ) é contraindicação para trombólise. ${ }^{1}$

\section{Dissecção aórtica}

É fundamental iniciar imediatamente o tratamento para evitar extensão da dissecção e ruptura. O tratamento é direcionado para a diminuição da pressão arterial e da frequência cardíaca. ${ }^{1}$ É recomendada redução da PAS para níveis em torno de 100-120 mmHg ou o menor valor que permita a perfusão adequada de órgãos vitais. Deve ser administrado betabloqueador para manter a Fc em menos de 60 bpm, sendo que para diminuição rápida do estresse na parede da aorta devem ser administrados agentes intravenosos. Caso haja contraindi-

Tabela 1. Medicamentos usados por via parenteral para o tratamento das emergências hipertensivas

\begin{tabular}{|c|c|c|c|c|c|}
\hline $\begin{array}{l}\text { nitroprussiato de } \\
\text { sódio (vasodilatador } \\
\text { arterial e venoso) }\end{array}$ & $0,25-10 \mu \mathrm{g} / \mathrm{kg} / \mathrm{min} \mathrm{EV}$ & Imediato & $1-2 \mathrm{~min}$ & $\begin{array}{l}\text { Náuseas, vômitos, } \\
\text { intoxicação por cianeto. } \\
\text { Cuidado na insuficiência } \\
\text { renal e hepática e na } \\
\text { pressão intracraniana alta. } \\
\text { Hipotensão grave. }\end{array}$ & $\begin{array}{l}\text { maioria das } \\
\text { emergências } \\
\text { hipertensivas }\end{array}$ \\
\hline $\begin{array}{l}\text { nitroglicerina } \\
\text { (vasodilatador } \\
\text { arterial e venoso) }\end{array}$ & $5-100 \mu \mathrm{g} / \mathrm{min} \mathrm{EV}$ & $2-5 \min$ & $3-5 \min$ & $\begin{array}{l}\text { cefaleia, taquicardia reflexa, } \\
\text { taquifilaxia, flushing, meta- } \\
\text { hemoglobinemia }\end{array}$ & $\begin{array}{l}\text { insuficiência } \\
\text { coronariana, } \\
\text { insuficiência } \\
\text { ventricular esquerda }\end{array}$ \\
\hline $\begin{array}{l}\text { metoprolol } \\
\text { (bloqueador } \\
\text { beta-adrenérgico } \\
\text { seletivo) }\end{array}$ & $\begin{array}{l}5 \mathrm{mg} \text { EV (repetir } \\
\text { 10/10min se necessário } \\
\text { até } 20 \mathrm{mg} \text { ) }\end{array}$ & $5-10 \mathrm{~min}$ & $3-4 \mathrm{~h}$ & $\begin{array}{l}\text { bradicardia, bloqueio } \\
\text { atrioventricular avançado, } \\
\text { insuficiência cardíaca, } \\
\text { broncoespasmo }\end{array}$ & $\begin{array}{l}\text { insuficiência } \\
\text { coronariana, } \\
\text { dissecção aguda de } \\
\text { aorta (em combinação } \\
\text { com nitroprussiato) }\end{array}$ \\
\hline $\begin{array}{l}\text { esmolol } \\
\text { (bloqueador } \\
\text { beta-adrenérgico } \\
\text { seletivo de ação } \\
\text { ultrarrápida) }\end{array}$ & $\begin{array}{l}\text { ataque } 500 \mu \mathrm{g} / \mathrm{kg} \text {; } \\
\text { infusão intermitente } \\
25-50 \mu \mathrm{g} / \mathrm{kg} / \mathrm{min} \text {; } \\
\text { aumentar } 25 \mu \mathrm{g} / \mathrm{kg} / \\
\text { min a cada } 10-20 \mathrm{~min} \text {. } \\
\text { Máximo } 300 \mu \mathrm{g} / \mathrm{kg} / \mathrm{min}\end{array}$ & $1-2 \mathrm{~min}$ & $1-20 \mathrm{~min}$ & $\begin{array}{l}\text { náuseas, vômitos, BAV 10 } \\
\text { grau, espasmo brônquico, } \\
\text { hipotensão }\end{array}$ & $\begin{array}{l}\text { dissecção aguda de } \\
\text { aorta (em combinação } \\
\text { com nitroprussiato), } \\
\text { hipertensão pós- } \\
\text { operatória grave }\end{array}$ \\
\hline $\begin{array}{l}\text { fentolamina } \\
\text { (bloqueador alfa- } \\
\text { adrenérgico) }\end{array}$ & $\begin{array}{l}\text { infusão contínua 1-5 } \\
\text { mg; máximo } 15 \text { mg. }\end{array}$ & $1-2 \mathrm{~min}$ & $3-5 \mathrm{~min}$ & $\begin{array}{l}\text { taquicardia reflexa, flushing, } \\
\text { tontura, náuseas, vômitos }\end{array}$ & $\begin{array}{c}\text { excesso de } \\
\text { catecolaminas }\end{array}$ \\
\hline diltiazem & $\begin{array}{l}0,25-0,35 \mathrm{mg} / \mathrm{kg} \text { em } \\
\text { bolus e } 5-20 \mathrm{mg} / \mathrm{h} \mathrm{em} \\
\text { infusão contínua }\end{array}$ & $\begin{array}{l}1-3 \\
\text { minutos }\end{array}$ & $1-3$ horas & bradicardia & $\begin{array}{l}\text { As mesmas dos } \\
\text { betabloqueadores } \\
\text { em casos de } \\
\text { contraindicação ao } \\
\text { uso dos mesmos }\end{array}$ \\
\hline verapamil & $0,075-0,15 \mathrm{mg} / \mathrm{kg}$ & $3-5 \min$ & $30 \mathrm{~min}-6 \mathrm{~h}$ & bradicardia & $\begin{array}{l}\text { As mesmas dos } \\
\text { betabloqueadores } \\
\text { em casos de } \\
\text { contraindicação ao } \\
\text { uso dos mesmos }\end{array}$ \\
\hline
\end{tabular}

Características farmacológicas, efeitos colaterais, precauções e indicações dos principais anti-hipertensivos parenterais utilizados nas emergências hipertensivas. ${ }^{3,5}$ 
cação ao uso de betabloqueadores devem ser administrados bloqueadores de canais de cálcio como verapamil e diltiazem. ${ }^{9}$ Qualquer forma de tratamento que diminua a pressão mas promova taquicardia reflexa deve ser evitado. ${ }^{1}$ Nitroprussiato quando utilizado sozinho pode levar propagação da dissecação, devendo ser utilizado apenas junto com betabloqueadores. ${ }^{9}$

\section{Emergências neurológicas}

\section{Encefalopatia hipertensiva}

Ocorre quando a pressão arterial se eleva a ponto de sobrecarregar o mecanismo de autorregulação cerebral, podendo ocorrer cegueira, convulsões, coma e morte. Achados patológicos incluem disfunção endotelial, edema cerebral, hemorragias petequiais e microinfartos. Os pacientes tipicamente têm hipertensão arterial crônica não controlada e desenvolvimento lento de sintomas neurológicos, principalmente cefaleia, 48-72 horas antes. Os pacientes com sintomas neurológicos devem realizar exames de imagem para excluir acidente vascular cerebral isquêmico ou hemorrágico, já que os alvos de controle de pressão arterial diferem entre as duas patologias. ${ }^{1}$ Deve-se reduzir a pressão arterial em torno de $20 \%{ }^{6}$

\section{Acidente vascular cerebral isquêmico}

A elevação da pressão arterial é comum nestes pacientes, sendo que tanto hipotensão como hipertensão têm efeitos deletérios. Para cada aumento em $10 \mathrm{mmHg}$ acima de $180 \mathrm{mmHg}$ há $40 \%$ de risco de piora do estado neurológico. ${ }^{1} \mathrm{~A}$ elevação da pressão arterial pode ser secundária ao estresse do evento cerebral ou relacionada à náusea, dor, hipertensão preexistente, uma resposta fisiológica à hipóxia ou resposta a aumento da pressão intracraniana. Na maioria dos pacientes ocorre diminuição da pressão arterial nas primeiras horas após o acidente vascular sem tratamento específico. A pressão diminui espontaneamente quando o paciente vai para um quarto silencioso, é colocado em repouso, tem a bexiga esvaziada ou a dor é controlada. Além disso, o tratamento da hipertensão intra- craniana também pode levar à diminuição da pressão arterial. ${ }^{10}$

As razões teóricas pra baixar a pressão arterial incluem redução da formação de edema cerebral, diminuição do risco de transformação hemorrágica, prevenção de dano vascular futuro e prevenção de acidente vascular recorrente precoce. Além disso, terapia anti-hipertensiva urgente pode ser requerida em pacientes que se apresentam com encefalopatia hipertensiva, dissecção aórtica, insuficiência renal aguda, edema agudo de pulmão ou infarto agudo do miocárdio. Porém, o tratamento agressivo pode levar à piora neurológica por reduzir a pressão de perfusão em áreas isquêmicas do cérebro. ${ }^{10}$ Isso ocorre porque após a instalação da isquemia há uma área densa central de tamanho variável e uma área ao redor com isquemia menos intensa, que pode ser salva, denominada penumbra isquêmica. Esta área de tecido atordoado, porém viável, depende de fluxo sanguíneo contínuo e altas pressões para manutenção da perfusão. ${ }^{1}$

$\mathrm{Na}$ ausência de consenso, a opinião do guideline publicado pela American Heart Association/American Stroke Association é que não devem ser administrados agentes antihipertensivos a menos que a PAD esteja acima de $120 \mathrm{mmHg}$ e a PAS acima de $220 \mathrm{mmHg}$. Quando o tratamento é indicado, a redução da pressão arterial deve ser feita com cautela. Um objetivo razoável é diminuir a pressão arterial em $15-25 \%$ no primeiro dia. ${ }^{10}$

Se houver deterioração clínica com redução da pressão, níveis mais elevados devem ser aceitos. ${ }^{1}$ Em pacientes potencialmente elegíveis à terapia de reperfusão, está recomendado tratamento para PAS acima de $185 \mathrm{mmHg}$ e PAD acima de $110 \mathrm{mmHg}$. Não deve ser realizada trombólise se a pressão arterial permanecer acima de 185-110 mmHg. ${ }^{1}$ Em pacientes que recebam rtPA o controle pressórico é essencial antes, durante e após a administração para evitar a ocorrência de transformação hemorrágica. ${ }^{10}$

Alguns pacientes têm hipertensão arterial crônica e necessitarão de reintrodução dos medicamentos. Os dados são limitados para avaliar 
o melhor momento de reintrodução, sendo que a determinação deste tempo depende de fatores como o estado neurológico, do mecanismo causador do AVC, da habilidade do paciente em engolir as medicações e da presença de doenças concomitantes. A maioria dos pacientes com AVC leve a moderado sem risco de hipertensão intracraniana pode ter suas medicações introduzidas 24 horas após o evento vascular. ${ }^{10}$

Como o nitroprussiato pode elevar a pressão intracraniana, os fármacos mais amplamente utilizados são labetalol e bloqueadores de canais de cálcio de ação rápida, como nicardipina e clevidipina, ${ }^{5,710}$ agentes indisponíveis no Brasil, onde amplamente ainda se utiliza o nitroprussiato.

\section{Hemorragia intracerebral}

A PA está frequentemente elevada em pacientes com hemorragia intracraniana aguda, geralmente diminuindo espontaneamente após alguns dias. Mecanismos potenciais incluem ativação do sistema neuroendócrino por estresse (sistema nervoso simpático, sistema renina-angiotensina, glicocorticoides) e aumento da pressão intracraniana. A hipertensão pode contribuir para a expansão do hematoma, do edema ao redor do hematoma e para novo sangramento, fatores que podem levar a pior prognóstico. ${ }^{11}$

As recomendações do guideline publicado pela American Heart Association/American Stroke Association são as seguintes:

- Tratamento agressivo com administração de infusão contínua de anti-hipertensivo se PAS maior que $200 \mathrm{mmHg}$ ou PAM maior que 150 mmHg; ${ }^{11}$

- Em pacientes com PAS maior que 180 $\mathrm{mmHg}$ e PAM maior que $130 \mathrm{mmHg}$ e possibilidade de elevação da pressão intracraniana deve ser considerada aferição da pressão intracraniana e uso de medicações intravenosas para manter a pressão de perfusão cerebral maior ou igual a $60 \mathrm{mmHg},{ }^{11}$
- $\quad$ Em pacientes com PAS maior que 180 $\mathrm{mmHg}$ e PAM maior que $130 \mathrm{mmHg}$ sem evidências de elevação da pressão intracraniana deve ser considerada redução modesta da pressão arterial para PAM em torno de $110 \mathrm{mmHg}$ e PAS em torno de $160 \mathrm{mmHg}$, reexaminando o paciente a cada 15 minutos. ${ }^{11}$

\section{Hipertensão perioperatória}

Pressão arterial descontrolada pode induzir novas lesões de órgãos-alvo, aumentar o risco de deiscência de suturas vasculares e sangramento, piorando o prognóstico. A hipertensão aguda pós-operatória se inicia duas a seis horas após a cirurgia e pode persistir por 24-48 horas. O período pós-operatório imediato (em torno de 2 horas) representa um período de instabilidade hemodinâmica significativa, sendo que a pressão arterial pode variar muito por ação de catecolaminas. Infusões intravenosas ou minibolus permitem melhor controle da pressão arterial. Nitroprussiato é efetivo na maioria das situações. Nitroglicerina também é benéfica. ${ }^{1}$

\section{Hipertensão por aumento de catecolaminas}

Nestes pacientes - por exemplo, portadores de feocromocitoma ou usuários de cocaína - betabloqueadores podem piorar a hipertensão devido à vasoconstrição periférica. Fentolamina é uma melhor escolha. ${ }^{8}$

\section{Eclampsia}

O fármaco de escolha para controle pressórico na eclampsia é a hidralazina, pois não é maléfica ao feto. Somente está autorizado o uso de nitroprussiato de sódio em casos nos quais o parto é iminente e não se consegue fazer o controle pressórico com hidralazina por via venosa. O objetivo deve ser manter a PAS entre 140 e $160 \mathrm{mmHg}$ e a PAD entre 90 e 105 mmHg. ${ }^{6}$ 


\section{Complicações do tratamento da hipertensão}

Apesar da necessidade do controle imediato da hipertensão em alguns casos, como descrito acima, algumas complicações do tratamento podem ocorrer, como:

hipotensão: por infusão rápida dos medicamentos, duração prolongada do efeito anti-hipertensivo, efeito aditivo de medicamentos, doença cardíaca nova, depleção de volume; ${ }^{1}$

piora do estado neurológico: isquemia secundária à hipotensão, encefalopatia hipertensiva, aumento da pressão intracraniana, efeito colateral das medicações, toxicidade por tiocianato, alterações metabólicas; ${ }^{1}$

piora de hipertensão: sobrecarga de volume, pseudotolerância, hipertensão secundária, esquema anti-hipertensivo fraco, baixa adesão; ${ }^{1}$

acidose metabólica: toxicidade por cianeto, hipoperfusão tecidual secundária; ${ }^{1}$

piora da função renal: hipoperfusão, depleção de volume, necrose tubular aguda. ${ }^{1}$

\section{Conclusão}

O reconhecimento imediato, avaliação e tratamento apropriados são condições fundamentais para diminuição da morbidade associada às emergências hipertensivas. Estes pacientes requerem imediata redução da pressão arterial para prevenir e controlar lesões de órgãosalvo, sendo que o melhor cenário para o controle da pressão arterial é o ambiente de terapia intensiva, com o uso de agentes intravenosos tituláveis. A abordagem apropriada de cada paciente depende da apresentação clínica. Devido à escassez de dados que apoiem um anti-hipertensivo em particular em relação a outro, a escolha e os objetivos do tratamento são baseados em consensos e devem ser guiados pela etiologia da hipertensão, extensão das lesões de órgãos-alvo e perfil hemodinâmico individual do paciente.

\section{Referências}

1. Scirica BM, Heyka RJ.Evaluation and Management of Hypertension in the Intensive Care Unit. In: Irwin RS, Rippe JM, editors. Irwin and Rippe's Intensive Care Medicine. Philadelphia: 2011. p.373-80.

2. Victor RG. Systemic Hypertension: Mechanisms and Diagnosis. In: Bonow RO, Mann DL, Zipes DP, Libby P, editors. Braunwald's Heart Disease. Philadelphia: 2012. p.952-3.

3. Sociedade Brasileira de Cardiologia / Sociedade Brasileira de Hipertensão / Sociedade Brasileira de Nefrologia. VI Diretrizes Brasileiras de Hipertensão. Arq Bras Cardiol. 2010;5(1 supl.1):1-51.

4. Seet RCS, Lim ECH. Hypertensive Brainstem Encephalopathy. Circulation. 2007;115:e310-1.

5. Marika PE, Rivera R. Hypertensive emergencies: an update. Curr Opin Crit Care. 2011;17:569-80.

6. Feitosa-Filho GS, Lopes RD, Poppi NT, et al. Emergências hipertensivas. Rev Bras Ter Intensiva. 2008;20(3):305-12.

7. Kaplan NM. Systemic Hypertension: Therapy. In: Bonow RO, Mann DL, Zipes DP, Libby $P$, editors. Braunwald's Heart Disease. Philadelphia: 2012. p.970-1.

8. Sutters M. Systemic Hypertension. In: McPhee SJ, Papadakis MA, editors. Current Medical Diagnosis and Treatment. California: 2009. p.411-4.

9. Braverman AC, Thompson RW, Sanchez LS. Diseases of the Aorta. In: Bonow RO, Mann DL, Zipes DP, Libby P, editors. Braunwald's Heart Disease. Philadelphia: 2012. p.1326-7.

10. American Heart Association/American Stroke Association. Guidelines for the Early Management of Adults with Ischemic Stroke. Stroke. 2007;38:1655-711.

11. American Heart Association/American Stroke Association. Guidelines for the Management of Spontaneous Intracerebral Hemorrhage. Stroke. 2010;41:2108-29. 